\title{
Sphenoid bone metastasis in lung cancer: An unusual finding
}

Sara Braga ${ }^{1}$, João F. Costa ${ }^{1}$, Gonçalo Santos ${ }^{2}$

A 68-year-old man with a recent diagnosis of small cell lung carcinoma, presented for a routine evaluation at our local lung cancer department, aiming to start treatment with carboplatin and etoposide. During the evaluation he seemed disoriented and complained of a headache. On physical examination only spatiotemporal disorientation stood out on the neurologic examination, with no other changes to report. Blood tests revealed hyponatremia, and elevated creatinine, alkaline phosphatase, gamma-GT, bilirubin, $C$ reactive protein and procalcitonin. He was then admitted and treated for possible urinary infection, later confirmed by positive urine and blood cultures for Escherichia coli. Considering maintained symptoms, he was further investigated with a head CT scan with contrast to rule out brain metastases and it end up revealing a sphenoidal osteolytic lesion measuring $20 \times 20 \times 14$ $\mathrm{mm}$ (Figure $1 \mathrm{~A}$ ), in addition to other smaller cranial bone metastases. Head MRI was also requested to allow a better characterization, showing an osteolytic lesion centered on the right paramedian region of the basisphenoid, with pronounced hyperintensity on T2-FLAIR images (Figure 1B), and homogeneous signal enhancement after contrast administration (Figures 1C and 1D).

The case presented poses an unusual finding. Although imaging study was performed to clarify the presence of brain metastases, the sphenoid metastasis

\author{
AFFILIATION \\ 1 Department of Pulmonology, Unidade Local \\ de Saúde da Guarda E.P.E. - Hospital Sousa \\ Martins, Portugal \\ 2 Imaging Department, Unidade Local de \\ Saúde da Guarda E.P.E. - Hospital Sousa \\ Martins, Portugal
}

\section{CORRESPONDENCE TO}

Sara Braga. Department of Pulmonology, Unidade Local de Saúde da Guarda E.P.E.

- Hospital Sousa Martins, Avenida Rainha D. Amélia 19, 6300-035 Guarda, Portugal. E-mail: sara_bmachado@hotmail.com ORCID ID: https://orcid.org/0000-0003-1066$767 x$

\section{KEYWORDS}

lung cancer, metastasis, sphenoid bone, bone metastasis

Received: 18 September 2021

Revised: 22 October 2021

Accepted: 21 November 2021

Figure 1. Head CT (A), axial plane: sphenoidal osteolytic lesion measuring $20 \times 20 \times 14 \mathrm{~mm}$. Head MRI, axial T2 FLAIR-weighted images (B): osteolytic lesion centered on the right paramedian region of the basisphenoid, with pronounced hyperintensity. Head MRI, T1-weighted sagittal images, before (C) and after (D) gadolinium contrast administration: homogeneous enhancement of the lesion
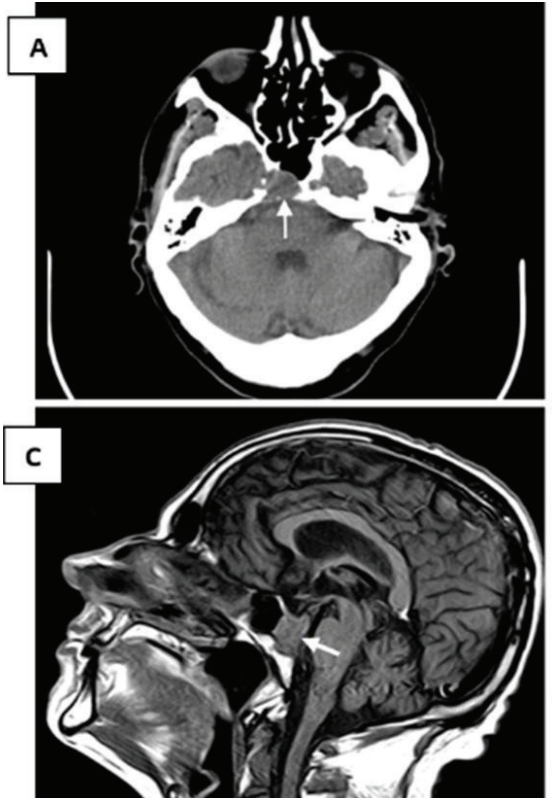
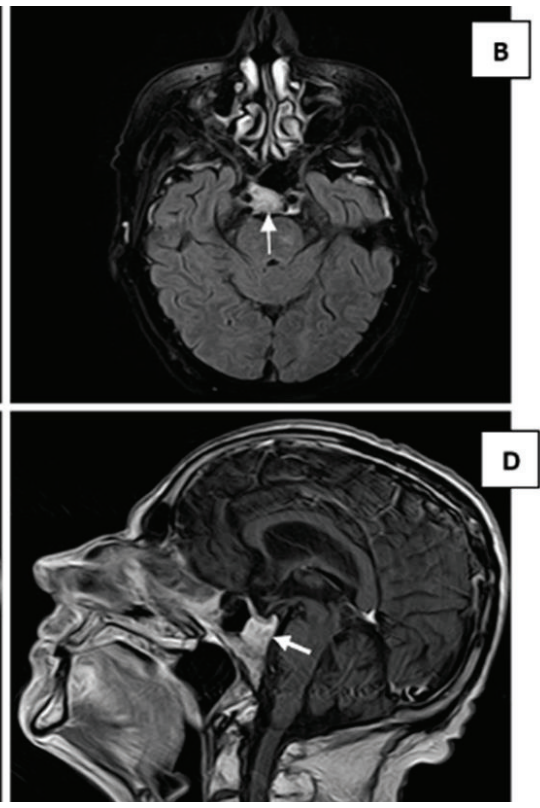
was not expected, and it was found by chance. Patients with lung cancer are at a higher risk of developing bone metastases (around 40\%), but sphenoid bone metastasis is rarely seen ${ }^{1-3}$.

Symptoms resulting from metastatic compression and compression of cranial nerves (such as ocular symptoms, headache, and facial dysesthesia) may occur and the physician should always be aware of them ${ }^{2,4}$.

The patient eventually passed away after a second cycle of chemotherapy, due to deterioration of the general condition, with worsening of renal function, anemia and grade I mucositis.

\section{CONFLICTS OF INTEREST}

The authors have completed and submitted the ICMJE Form for Disclosure of Potential Conflicts of Interest and none was reported.

\section{FUNDING}

There was no source of funding for this research.

\section{ETHICAL APPROVAL AND INFORMED CONSENT}

Ethical approval was obtained from Unidade Local de Saúde da Guarda, and informed consent was provided by the participant.

\section{DATA AVAILABILITY}

The data supporting this research are available from the author on reasonable request.

\section{PROVENANCE AND PEER REVIEW}

Not commissioned; externally peer reviewed.

\section{REFERENCES}

1. Turner RC, Lucke-Wold BP, Hwang R, Underwood BD. Lung cancer metastasis presenting as a solitary skull mass. J Surg Case Rep. 2016;2016(6):rjw116. doi:10.1093/jscr/rjw116

2. Bandoh $\mathrm{N}$, Ichikawa $\mathrm{H}$, Asahi $\mathrm{A}$, et al. Lung Adenocarcinoma with Cheek Dysesthesia as an Initial Symptom: A Case Report and Literature Review. Case Rep Oncol. 2019;12(2):650-656. doi:10.1159/000502053

3. Gupta PK, Mital M, Dwivedi A, Gupta K. Metastasis of greater wing of sphenoid bone in bronchogenic carcinoma: A unusual case report. J Cancer Res Ther. 2011;7(2):195-197. doi:10.4103/0973-1482.82921

4. Fukai S, Okabe N, Mine H, Takagi H, Suzuki H. Garcin syndrome caused by sphenoid bone metastasis of lung cancer: a case study. World J Surg Oncol. 2018;16(1):46. doi:10.1186/s12957-018-1351-4 